Pathophysiology

of Haemostasis and Thrombosis
Pathophysiol Haemost Thromb 2007-08;36:266-270

DOI: $\underline{10.1159 / 000252823}$
Received: April 6, 2009

Accepted after revision: September 9, 2009

\title{
Granulocyte Colony-Stimulating Factor Increases the Platelet Volume in Peripheral Stem Cell Apheresis Donors
}

\author{
Akihiro Ihara $^{a}, b$ Keiko Matsui ${ }^{c}$ Ryouta Minami ${ }^{c}$ Shuzou Uchida ${ }^{c}$ Shuji Ueda ${ }^{c}$ \\ Tetsuo Nishiurac \\ ${ }^{a}$ Department of Hematology, Fukuyama Chuo Hospital, and ${ }^{b}$ Institute of Clinical Research and ${ }^{\mathrm{C}}$ Department of \\ Hematology and Oncology, National Hospital Organization Kure Medical Center and Chugoku Cancer Center, \\ Hiroshima, Japan
}

\section{Key Words}

Granulocyte colony-stimulating factor $\cdot$ Mean

platelet volume Allogeneic peripheral blood stem

cell transplantation donor

\begin{abstract}
We investigated the short-term influence of granulocyte colony-stimulating factor (G-CSF) administration on platelet counts and platelet indices in 12 donors $(8$ males and $4 \mathrm{fe}-$ males; median age 34 years, range 16-49) for peripheral stem cell transplantation using an automated blood cell analyzer. On day 3 (D3) compared with D0, 11 donors with normal laboratory and physical findings showed increases in platelet indices $\left(\chi^{2}=12.0, p=0.0025\right)$. Furthermore, mean platelet volume (MPV) was significantly increased $(p=0.04)$. Also, platelet count decreased, and platelet distribution width and platelet-large cell ratio were increased, but these were not significant. On the contrary, 1 donor with abnormal laboratory findings who had large platelets (MPV $11.4 \mathrm{fl}$ ) before G-CSF administration showed decreases in platelet indices (MPV $10.3 \mathrm{fl}$ ) on D3, although platelet count $(18.2 \times$ $10^{4} / \mu \mathrm{l}$ ) decreased after G-CSF administration. G-CSF administration induces an inflammatory process with endothelial
\end{abstract}

cell activation. This is probably the reason why platelet volume increases after G-CSF use. This is the first report showing that G-CSF administration immediately induces increases in large platelets in peripheral stem cell transplant donors before harvest.

Copyright $\odot 2009$ S. Karger AG, Basel

\section{Introduction}

Granulocyte colony-stimulating factor (G-CSF) is widely used in healthy donors for allogeneic peripheral blood stem cell transplantation. However, some cases of thrombosis occurring in elderly donors, possibly because of G-CSF, have also been published $[1,2]$. Some data have suggested that G-CSF induces a hypercoagulable state according to the results of platelet aggregation, platelet activation markers and coagulation studies $[3,4]$. The mechanism of the effect of G-CSF on platelet activation remains poorly understood. However, platelet activation may cause an accelerated platelet turnover and an increase in larger platelets. Recent studies have shown that an increase in large platelets is a risk factor for acute coronary syndromes $[5,6]$ and cerebral infarction $[7,8]$.

\section{KARGER}

Fax +41613061234

E-Mail karger@karger.ch

www.karger.com (c) 2009 S. Karger AG, Basel

$1424-8832 / 08 / 0365-0266 \$ 24.50 / 0$

Accessible online at:

www.karger.com/pht
Akihiro Ihara

Department of Hematology

Fukuyama Chuo Hospital

Hiroshima (Japan)

Tel. +81 84922 0800, Fax +81 84926 7074, E-Mail a-ihara@ ken-o-gr.jp 
Table 1. Minimum, maximum and median platelet counts and platelet indices in 11 normal donors

\begin{tabular}{llllr}
\hline & PLT,$\times 10^{4} / \mu \mathrm{l}$ & MPV, $\mathrm{fl}$ & P-LCR, fl & \multicolumn{1}{l}{ PDW, fl } \\
\hline D0 & $15.3-34.1(24.5)$ & $8.1-10.9(10.0)$ & $12.6-31.8(26.6)$ & $9.7-12.8(12.3)$ \\
D1 & $15.9-30.2(23.2)$ & $8.2-11.2(10.0)$ & $13.1-34.2(24.3)$ & $9.7-13.0(11.0)$ \\
D2 & $17.4-27.9(22.0)$ & $9.6-11.4(10.1)$ & $21.0-29.6(26.0)$ & $10.3-14.1(12.3)$ \\
D3 & $16.4-25.3(21.4)$ & $9.5-11.2(10.4)^{*}$ & $20.3-35.6(29.1)$ & $10.7-14.3(12.9)$ \\
\hline
\end{tabular}

Figures in parentheses are medians. ${ }^{*} \mathrm{p}=0.04$.

We hypothesized that platelet size will increase on platelet activation after G-CSF use. To test this hypothesis, we investigated the short-term influence of G-CSF administration on platelet counts and platelet indices in 12 donors for stem cell transplantation using an automated blood cell analyzer.

\section{Subjects and Methods}

12 donors (8 males and 4 females; median age 34 years, range 16-49) were included, comprising 4 sons, 6 daughters, 1 mother and 1 brother. Human leukocyte antigen types were all mismatched, except for 1 brother. There was no medical history of note in any donors. Before G-CSF administration, medical history was taken, and physical examination and routine laboratory investigations (liver functions and serum lipids) were performed in all donors. As a result, 1 donor (a 27-year-old male) showed many abnormal findings. His body weight was $75 \mathrm{~kg}$, height was $160 \mathrm{~cm}$, and blood pressure was 160/95 mm Hg. Abdominal echography clearly revealed a fatty liver. Liver functions and other laboratory data were as follows: serum glutamic-oxaloacetic transaminase $27 \mathrm{IU} / \mathrm{l}$, serum glutamic-pyruvic transaminase 46 IU/l, uric acid $7.6 \mathrm{mg} / \mathrm{dl}$, total cholesterol $230 \mathrm{mg} / \mathrm{dl}$, triglycerides $222 \mathrm{mg} / \mathrm{dl}$, and C-reactive protein $0.05 \mathrm{mg} / \mathrm{dl}$. Electrocardiography and urinary examinations were normal. We explained to him the danger of G-CSF administration. The other 11 donors showed completely normal results for the above examinations. Therefore, we segregated this donor from the other 11 donors and evaluated him separately. None had taken drugs including aspirin or aspirin-containing drugs prior to donation. Written informed consent was obtained from each individual before starting the procedure.

G-CSF (lenograstin, Chugai Ltd., Tokyo, Japan, or filgrastin, Kirin Ltd., Tokyo, Japan) was given subcutaneously at a dose of $500 \mu \mathrm{g} /$ day to 6 donors (lenograstin) and $600 \mu \mathrm{g} /$ day to 6 donors (filgrastin) in 2 daily divided doses for 4 or 5 days. Stem cell apheresis procedures were started in 11 donors on the 4 th and in 1 donor on the 5th day after G-CSF administration. Platelet indices were measured before and after G-CSF administration before apheresis. Four blood samples were drawn at different times: day 0 (D0), within $2 \mathrm{~h}$ before G-CSF administration; D1,

G-CSF Increases the Platelet Volume in Peripheral Stem Cell Apheresis Donors at $24 \mathrm{~h}$; D2, after 2 days; and D3, 3 days after the first G-CSF administration. Venous blood samples were drawn by venipuncture of the forearm with minimum stasis through a 21gauge needle into sterile evacuated tubes containing 2K-EDTA. White blood cell, red blood cell and platelet counts, platelet indices (mean platelet volume, MPV; platelet-large cell ratio, $\mathrm{P}$ LCR; platelet distribution width, PDW), hemoglobin level and hematocrit were determined using an automated cell counter (Sysmex NE-8000, Sysmex Ltd., Kobe, Japan) within 2 h after venipuncture.

Fisher's least significant difference post hoc test was used to identify on which day group differences in the 11 normal donors accounted for significant $p$ values. The $\chi^{2}$ test was used to assess the 11 donors with normal and the 1 donor with abnormal physical and laboratory data.

\section{Results}

Changes observed in platelet counts and platelet indices in the donors after G-CSF administration are presented in figure 1. Minimum, maximum and median platelet counts and platelet indices of the 11 normal controls are also shown in table 1 . Their significance from D0 to D3 is shown in table 2. On D3 compared with D0, 11 of 12 donors showed decreases in platelet count, excluding 1 donor with normal laboratory findings. Also, all 11 donors with normal laboratory and physical findings showed increases in platelet indices $\left(\chi^{2}=12.0, \mathrm{p}=\right.$ 0.0025). Furthermore, on D3 compared with D0, MPV was significantly increased $(\mathrm{p}=0.04)$, platelet count was decreased, and PDW and P-LCR were increased, but non-significantly (tables 1, 2). On the contrary, the donor with abnormal laboratory findings who showed large platelets (MPV $11.4 \mathrm{fl}, \mathrm{P}-\mathrm{LCR} 36.4 \%$ and PDW $14.9 \mathrm{fl}$ ) before G-CSF administration showed decreases in platelet indices (MPV $10.3 \mathrm{fl}, \mathrm{P}-\mathrm{LCR} 28.9 \%$ and PDW $13.5 \mathrm{fl}$ ) on D3, although the platelet count $\left(18.2 \times 10^{4} / \mu \mathrm{l}\right)$ decreased after G-CSF administration (fig. 1). There were 

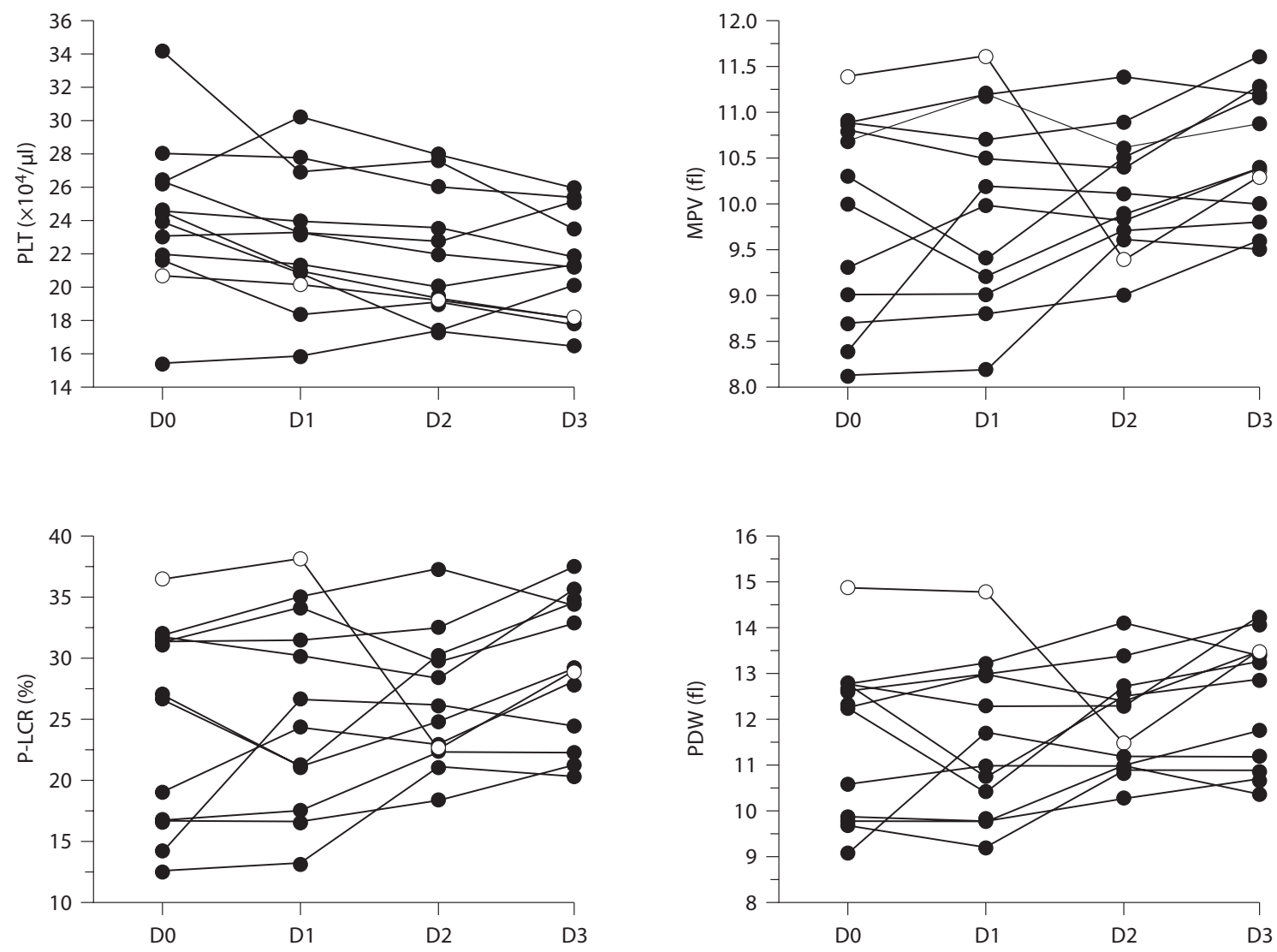

Fig. 1. Time course of platelet counts and platelet indices after G-CSF administration in allogeneic transplant donors. P-LCR is the percentage of large platelets defined as the platelet volume exceeding $12 \mathrm{fl}$. $\bigcirc=$ The case with abnormal laboratory and physical findings; $\bullet=11$ cases showing normal laboratory and physical findings.

Table 2. $\mathrm{p}$ values on D0, D1, D2 and D3 for platelet counts and platelet indices in 11 normal donors

\begin{tabular}{lllllll}
\hline & D0 vs. D1 & D0 vs. D2 & D0 vs. D3 & D1 vs. D2 & D1 vs. D3 & D2 vs. D3 \\
\hline PLT & 0.38 & 0.16 & 0.09 & 0.59 & 0.39 & 0.74 \\
MPV & 0.76 & 0.25 & 0.04 & 0.41 & 0.08 & 0.34 \\
P-LCR & 0.93 & 0.29 & 0.08 & 0.25 & 0.07 & 0.48 \\
PDW & 0.70 & 0.29 & 0.07 & 0.50 & 0.14 & 0.41 \\
\hline
\end{tabular}


no differences in platelet counts and platelet indices between the groups using lenograstin and filgrastin. The procedure did not alter hemoglobin concentrations in the donors until D3.

\section{Discussion}

Decreases in platelet count immediately before apheresis (nonsignificant) and also decreased hemoglobin levels $(p<0.01)$ occurred following G-CSF administration [9]. However, we did not identify decreased hemoglobin levels. Decreases in platelet counts after G-CSF administration without apheresis have been reported [10]. A preapheresis platelet decrease was correlated with the total G-CSF dose administered, baseline platelet level and donor age [11]. There was a difference in the frequency of platelet reduction after G-CSF between our and the previous results. This may be due to a difference in donor ages or the development of atherosclerosis as a result of lipidemia or hypertension. Our donors were all young and showed normal laboratory and physiological findings, except for 1 donor who showed decreased platelet indices. Other studies included many aged donors with abnormal laboratory data or no laboratory examination data.

The mechanism of such thrombocytopenia is unclear. Platelets are directly influenced by G-CSF through the G-CSF receptor. This signal may cause platelet aggregation in the presence of agonists, including collagen and/ or ADP at local sites in the bloodstream. Granulocytes are also activated by G-CSF in vivo. The expression of adhesion molecules may modulate the function of platelets, monocytes and vascular endothelial cells and may enhance cell-to-cell attachment and interaction with co- agulation factors on the cell surface [12]. Thus, it may lead to a hypercoagulable state. Cella et al. [13] hypothesized that during G-CSF administration, a significant alteration of the endothelium might occur in association with abnormal oxidative stress. G-CSF administration induces an inflammatory process with endothelial cell activation.

We showed that the thrombin-antithrombin complex was positively correlated with platelet indices in patients with aortic aneurysm [14] and ischemic heart disease [15], and that von Willebrand factor antigen and age are also positively correlated with platelet indices in aged normal controls [14]. These findings indicate that thrombin generation due to endothelial degeneration in atherosclerotic diseases and increased von Willebrand factor antigen as a result of endothelial injury caused by aging may consume platelets and accelerate platelet turnover, and large platelets may increase in the peripheral blood. Platelet volume decreases in aortic aneurysms [14] and in ischemic heart disease patients with severe coronary artery disease [15]. The mechanism of platelet volume decrease after G-CSF use in our 27-year-old male with abnormal laboratory data may be the same as that of the above aortic aneurysm and ischemic heart disease patients. This is the first report showing that G-CSF administration immediately induces increases in MPV in peripheral stem cell donors after G-CSF administration, especially on D3.

\section{Conclusion}

Platelet volume increases immediately after G-CSF administration, especially on D3. This increase may be a risk factor for thrombosis.
References

G-CSF Increases the Platelet Volume in Peripheral Stem Cell Apheresis Donors
1 Anderlini P, Körbling M, Dale D, Gratwohl A, Schmitz N, Stroncek D, Howe C, Leitman S, Horowitz M, Gluckman E, Rowley S, Przepiorka D, Champlin R: Allogeneic blood stem cell transplantation: considerations for donors. Blood 1997;90:903-908.

2 Gutierrez-Delgado F, Bensinger W: Safety of granulocyte colony-stimulating factor in normal donors. Curr Opin Hematol 2001;8: 155-160.

-3 LeBlanc R, Roy J, Demers C, Vu L, Cantin G: A prospective study of G-CSF effects on hemostasis in allogeneic blood stem cell donors. Bone Marrow Transplant 1999;23:991996.

\footnotetext{
4 Kuroiwa M, Okamura T, Kanaji T, Okamura S, Harada M, Niho Y: Effects of granulocyte colony-stimulating factor on the hemostatic system in healthy volunteers. Int J Hematol 1996;63:311-316

5 Endler G, Klimesch A, Sunder-Plassmann $\mathrm{H}$, Schillinger M, Exner M, Mannhalter C, Jordanova N, Christ G, Thalhammer R, Huber K, Sunder-Plassmann R: Mean platelet volume is an independent risk factor for myocardial infarction but not for coronary artery disease. Br J Haematol 2002;117:399404.
} 
6 Ihara A, Kawamoto T, Matsumoto K, Shouno S, Hirahara C, Morimoto T, Noma Y: Relationship between platelet indexes and coronary angiographic findings in patients with ischemic heart disease. Pathophysiol Haemost Thromb 2006;35:376-379.

$>7$ GreiseneggerS, EndlerG, Hsieh K, Tentschert S, Mannhalter C, Lalouschek W: Is elevated mean platelet volume associated with a worse outcome in patients with acute ischemic cerebrovascular events? Stroke 2004;35: 1688-1691.

$>8$ Bath P, Algert C, Chapman N, Neal B: Association of mean platelet volume with risk of stroke among 3134 individuals with history of cerebrovascular disease. Stroke 2004;35: 622-626.
>9 Kuroiwa M, Okamura T, Kanaji T, Okamura S, Harada M, Niho Y: Effects of granulocyte colony-stimulating factor on the hemostatic system in healthy volunteers. Int J Hematol 1996;63:311-316.

10 Falanga A, Marchetti M, Evangelista V, et al: Neutrophil activation and hemostatic changes in healthy donors receiving granulocyte colony-stimulating factor. Blood 1999;93: 2506-2515.

11 Tassi C, Tazzari PL, Bonifazi F, Giudice V, Nannetti A, Ricci F, Rizzi S, Bandini G, Conte R: Short- and long-term haematological surveillance of healthy donors of allogeneic peripheral haematopoietic progenitors mobilized with G-CSF: a single institution prospective study. Bone Marrow Transplant 2005;36:289-294.

12 Declerck PJ, Alessi MC, Verstreken M, Kruithof EK, Juhan-Vague I, Collen D: Measurement of plasminogen activator inhibitor 1 in biologic fluids with a murine monoclonal antibody-based enzyme-linked immunosorbent assay. Blood 1988;71:220-225.
13 Cella G, Marchetti M, Bignoli A, Randi ML, Saggiorato G, Pasetto L, Pagnan A, Barbui T, Falanga A: Blood oxidative status and selectins plasma levels in healthy donors receiving granulocyte-colony stimulating factor. Leukemia 2006;20:1430-1434.

14 Ihara A, Matsumoto K, Kawamoto T, Shouno S, Kawamoto J, Katayama A, Yoshitatsu M, Izutani H: Relationship between hemostatic markers and platelet indices in patients with aortic aneurysm. Pathophysiol Haemost Thromb 2006;35:451-456.

15 Ihara A, Kawamoto T, Matsumoto K, Shouno S, Morimoto T, Noma Y: Relationship between hemostatic factors and the platelet index in patients with ischemic heart disease. Pathophysiol Haemost Thromb 2006;35: 388-391. 